\title{
Novel Sternal Protection Device for Cardiac Surgery Via Median Sternotomy Incision
}

\author{
Silvana F. Marasco, FRACS, * David C. McGiffin, FRACS, * Adam D. Zimmet, FRACS, * Pablo C. Solis, MBA, † \\ Judy M. Bingham, MHA, $\neq$ and Randall A. Moshinsky, FRACS\$//
}

Objective: Sternal bleeding during cardiac surgery is currently controlled using bone wax or other chemical substances that may result in adverse effects and affect wound healing and recovery. The purpose of this study was to identify a safe, cost-effective, and easy-to-use technique to reduce sternal bleeding and sternal trauma during cardiac surgery.

Methods: After sternotomy, a sternal protection device was placed over each hemisternal section before insertion of the retractor and remained in situ until the end of surgery. Sternal bleeding and ease of use were assessed and recorded during surgery. Sternal trauma was assessed and recorded within 5 minutes of removal of the device, and overall satisfaction (Global Impression) and any intraoperative adverse events or device malfunction were reported at surgery completion. Patients were followed up 24 hours and 4 weeks after surgery.

\section{Video clip is available online.}

Accepted for publication August 28, 2017.

From the *Department of Cardiothoracic Surgery, Alfred Hospital, Melbourne, Victoria, Australia; †Protego Medical Pty Ltd, Melbourne, Victoria, Australia; †Easington Pty Ltd, Camberwell, Victoria, Australia; §Department of Cardiothoracic Surgery, Monash Health, Clayton, Victoria, Australia; and ||Department of Surgery, School of Clinical Sciences at Monash Health, Monash University, Clayton, Victoria, Australia.

A video clip and supplemental digital content are available for this article. Direct URL citations appear in the printed text and are provided in the HTML and PDF versions of this article on the journal's Web site (www. innovjournal.com).

Supported by Protego Medical Pty Ltd, Melbourne, Victoria, Australia, that provided the devices and paid expenses associated with the conduct of the study.

Disclosures: Pablo C. Solis, MBA, is the CEO of Protego Medical Pty Ltd, Melbourne, Victoria, Australia; Judy M. Bingham, MHA, is a consultant for Protego Medical Pty Ltd and managed nonpatient care aspects of the clinical study. Randall A. Moshinsky, FRACS, is the inventor of the device, consultant, and shareholder in Protego Medical Pty Ltd. Silvana F. Marasco, FRACS, David C. McGiffin, FRACS, and Adam D. Zimmet, FRACS, declare no conflicts of interest.

Address correspondence and reprint requests to Randall A. Moshinsky, FRACS, Department of Cardiothoracic Surgery, Monash Health, 246 Clayton Rd, Clayton, 3168, Victoria, Australia. E-mail: randallmoshinsky@bigpond.com.

Copyright (C) 2017 The Author(s). Published by Wolters Kluwer Health, Inc. on behalf of the International Society for Minimally Invasive Cardiothoracic Surgery

This is an open-access article distributed under the terms of the Creative Commons Attribution-Non Commercial-No Derivatives License 4.0 (CCBY-NC-ND), where it is permissible to download and share the work provided it is properly cited. The work cannot be changed in any way or used commercially without permission from the journal.

ISSN: $1556-9845 / 17 / 1205-0370$
Results: Twelve patients completed the study. Adverse events reported were not considered related to the device. No sternal trauma was identified in any patient. In 9 of 11 patients, sternal bleeding was reduced after insertion of the device. The device was generally considered easy to use, although some difficulty was encountered when used with the Internal Mammary Artery retractor.

Conclusions: Our data suggest that the device is safe and able to reduce sternal bleeding during surgery using sternal retractors. We recommend further studies in a larger population of patients with a control group to evaluate the device's ability to reduce the morbidity associated with sternal bleeding and sternal trauma.

Key Words: Median sternotomy, Sternal protection device, Sternal retractor, IMA retractor.

\section{(Innovations 2017;12:370-374)}

C ternal retractors spread apart the left and right hemisternal $\checkmark$ sections to expose the pericardium and heart for cardiac surgery. The external force transmitted to the hemisternal sections through the retractor blades often results in trauma to the hemisternal sections, including sternal fractures ${ }^{1,2}$ with increase in postoperative pain, slower recovery ${ }^{3,4}$ and increased postoperative bleeding.

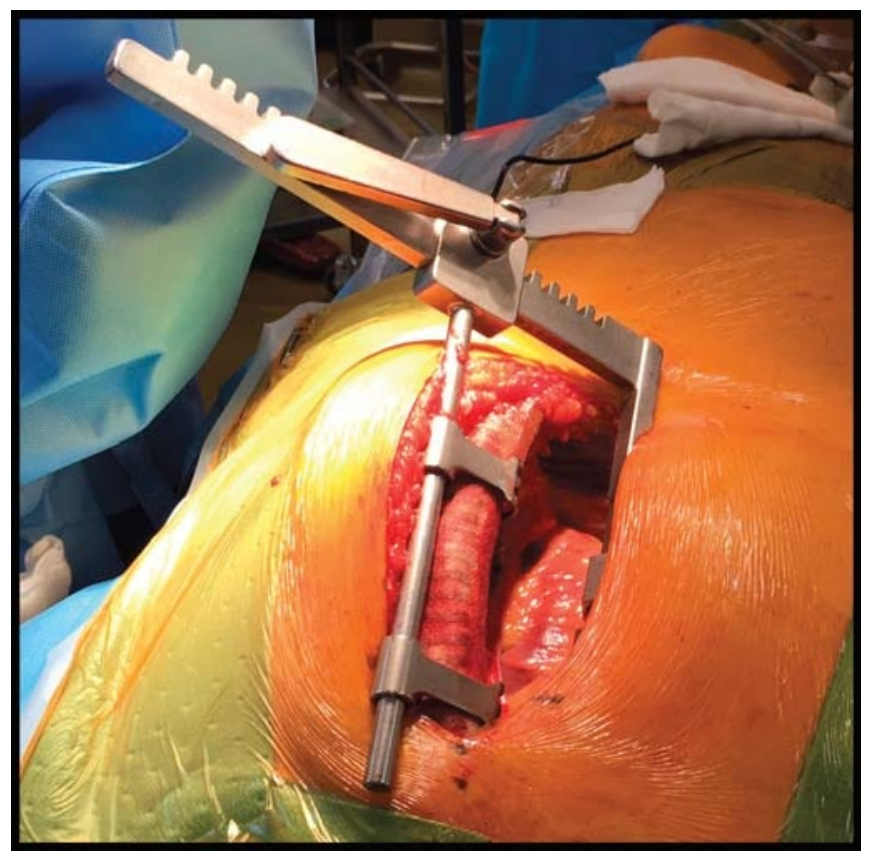

FIGURE 1. Sternal protection device in situ with an IMA retractor. 


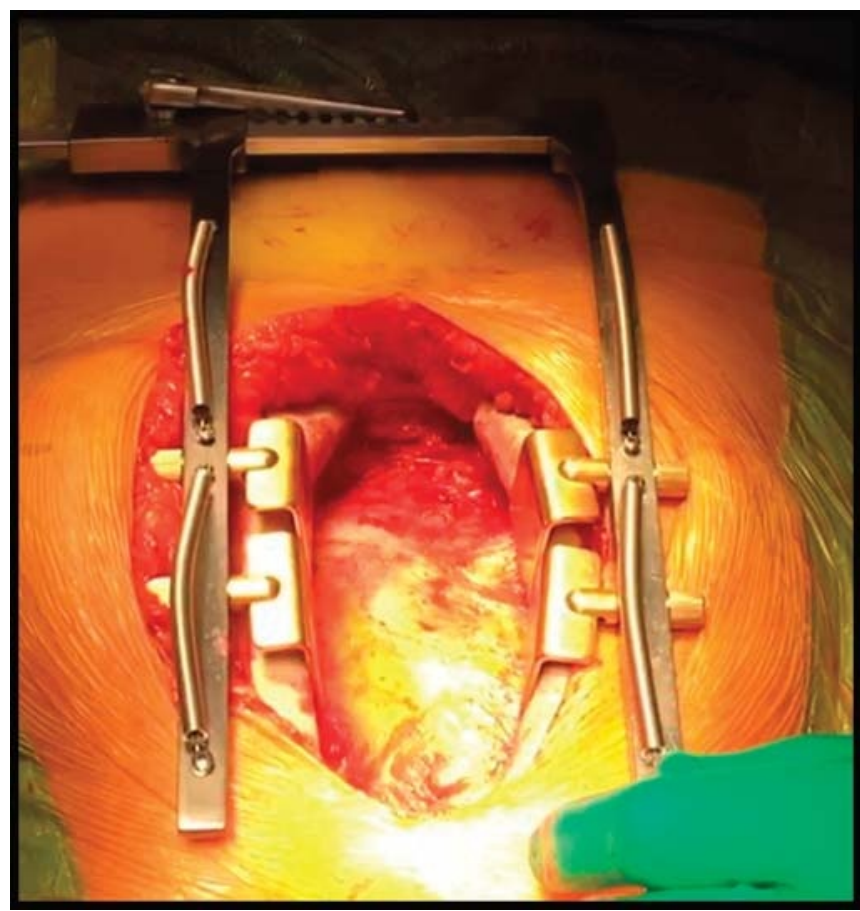

FIGURE 2. Sternal protection device in situ with a sternal retractor.

Bleeding from the cut hemisternal sections occurs after a median sternotomy incision and is difficult to control with diathermy. Traditional methods to reduce bleeding include the use of bone wax or other chemical substances such as fibrin sealant and microfibrillar collagen. Although cheap and quick to apply, bone wax is impossible to completely remove after surgery and degrades slowly, with potential adverse effects including inhibition of bone fusion and increased infection rates. ${ }^{3,6-10}$ Other chemical substances are costly. ${ }^{11}$ Because of increasing age, frailty, medical comorbidities including osteoporosis, and complexity of the surgery, increasing numbers of patients are at high risk for sternal instability and wound healing complications after cardiac surgery.
The aim of this open-label, noncomparative, single-center pilot study was to evaluate the safety of a novel sternal protection device (SPD), the ability of the device to protect the sternum from trauma and reduce blood loss, and the ease of use of the device.

\section{METHODS}

The study was approved by the human research ethics committee at the study site (Melbourne, Australia; Protocol Number 01025-00014; Project Number 4/15; April 01, 2015). Twelve patients (10 male, 2 female) with mean \pm SD age of $64.6 \pm 13.8$ years (range $=39.8-79.8)$ and mean \pm SD weight of $85.0 \pm 16.6 \mathrm{~kg}$ (range $=63.1-113.0)$ provided informed consent and participated in the study. Ten underwent coronary artery bypass graft $(\mathrm{CABG})$ surgery and 2 underwent aortic valve replacement surgery. The Delacroix-Chevalier Internal Mammary Artery (IMA) retractor (Delacroix-Chevalier, Paris, France) was used in all patients who underwent IMA harvest. For sternal retraction, the Ankeney Sternal Retractor (Symmetry Surgical Inc, Antioch, TN USA) or Favaloro-Morse Sternal Retractor (Teleflex Inc, Morrisville, NC USA) was used.

The Jupiter SPD (Protego Medical, Melbourne, Australia) is a sterile single-use device comprising 2 L-shaped components. The SPD comprises an inner stainless steel frame, covered by foam and fabric components. The device is able to conform to variations in sternal height and curvature. Insertion of the SPD and use with a sternal retractor are illustrated in the video (see Video, Supplemental Digital Content 1, http://links.lww. com/INNOV/A147). The SPD in situ with an IMA retractor is illustrated in Figure 1 and the SPD in situ with a sternal retractor is illustrated in Figure 2. Surgical instruments and equipment for cardiac surgical procedures were provided by the study site.

Patient suitability for the study was assessed at a screening visit within 3 weeks of surgery, and patients were followed up after surgery at 24 hours and 4 weeks.

For patients requiring CABG surgery using the IMA as a conduit, the SPD was placed over each hemisternal section after sternotomy and before insertion of the IMA retractor. For patients not requiring $\mathrm{CABG}$ surgery with the IMA as a conduit, the SPD was applied before insertion of the sternotomy retractor alone. The SPD is compatible with both IMA and sternotomy retractors.

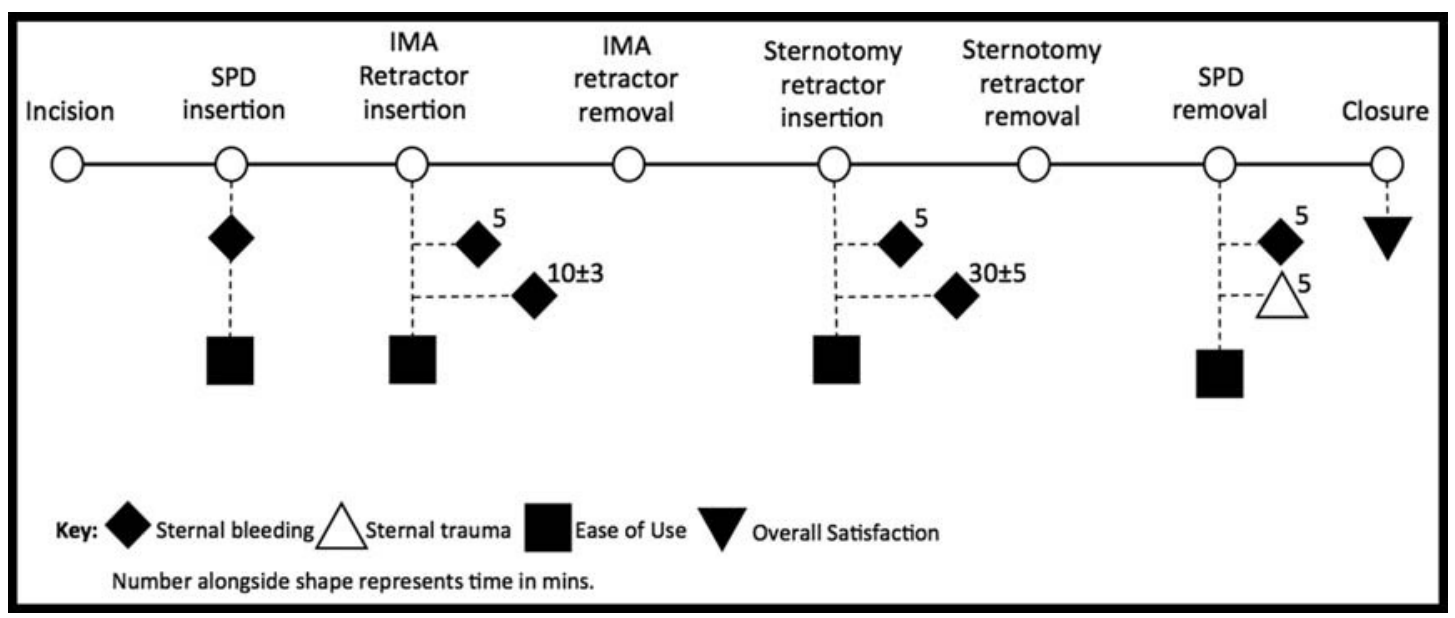

FIGURE 3. Assessment time points. 
TABLE 1. Sternal Bleeding Assessment

\begin{tabular}{|c|c|c|c|c|c|c|c|}
\hline \multirow[b]{2}{*}{$\mathbf{N}=12$} & \multirow[b]{2}{*}{ Sternal Bleeding } & \multirow{2}{*}{$\frac{\text { SPD Insertion }}{\text { Immediately Before }}$} & \multicolumn{2}{|c|}{ IMA Retractor Insertion } & \multicolumn{2}{|c|}{ Sternal Retractor Insertion } & \multirow{2}{*}{$\frac{\text { SPD Removal }}{\text { Within } 5 \text { min }}$} \\
\hline & & & Within 5 min* & $10 \pm 3 \mathrm{~min}^{*}$ & Within 5 min & $30 \pm 5 \mathrm{~min}$ & \\
\hline \multirow[t]{4}{*}{ IMA retractor and sternal retractor $(n=9)$} & None & 1 & 5 & 5 & 5 & 7 & - \\
\hline & Mild $\dagger$ & 3 & 1 & 1 & 3 & 2 & 4 \\
\hline & Moderate $\ddagger$ & 3 & - & - & 1 & - & 3 \\
\hline & Severe $\S$ & 2 & - & - & - & - & 2 \\
\hline \multirow[t]{4}{*}{ Sternal retractor only $(n=3)$} & None & - & - & - & 3 & 3 & - \\
\hline & Mild $\dagger$ & 1 & - & - & - & - & 1 \\
\hline & Moderate & 1 & - & - & - & - & - \\
\hline & Severe $\S$ & 1 & - & - & - & - & 2 \\
\hline
\end{tabular}

*Not recorded for 3 patients. $\uparrow$ Mild = intermittent drip or trickle of blood from sternum from 1 point only. $\$$ Moderate $=$ continuous drip or stream of blood from sternum from 1 point only. $\S$ Severe $=$ continuous stream of blood from more than 1 point.

IMA, internal mammary artery; SPD, sternal protection device.

No bone wax or other bone hemostatic agent was used in conjunction with the SPD, which remained in situ until the end of surgery.

During surgery, the surgeon assessed and recorded sternal bleeding at 6 time points and completed an ease of use assessment scale at 4 time points (Fig. 3). Sternal trauma was assessed within 5 minutes of removal of the SPD, and the Global Impression assessment scale (subjective assessment of overall satisfaction with the SPD) was completed. Any intraoperative adverse events (AEs) or device malfunction events were reported at the completion of surgery.

Safety assessments included AEs, device deficiencies, and concomitant medicines to treat AEs.

Because this was a proof-of-concept study, no formal statistical analysis was planned. Summary statistics included counts and percentages for categorical variables and the number of participants, mean, SD, median, minimum, and maximum for continuous variables.

\section{RESULTS}

The baseline bleeding rate immediately before SPD insertion was mild in $4(33 \%)$ of 12 patients, moderate in $4(33 \%)$ of 12 patients, and severe in 3 (25\%) of 12 patients. Only 1 patient was recorded as having no bleeding from the sternum at baseline (Table 1).

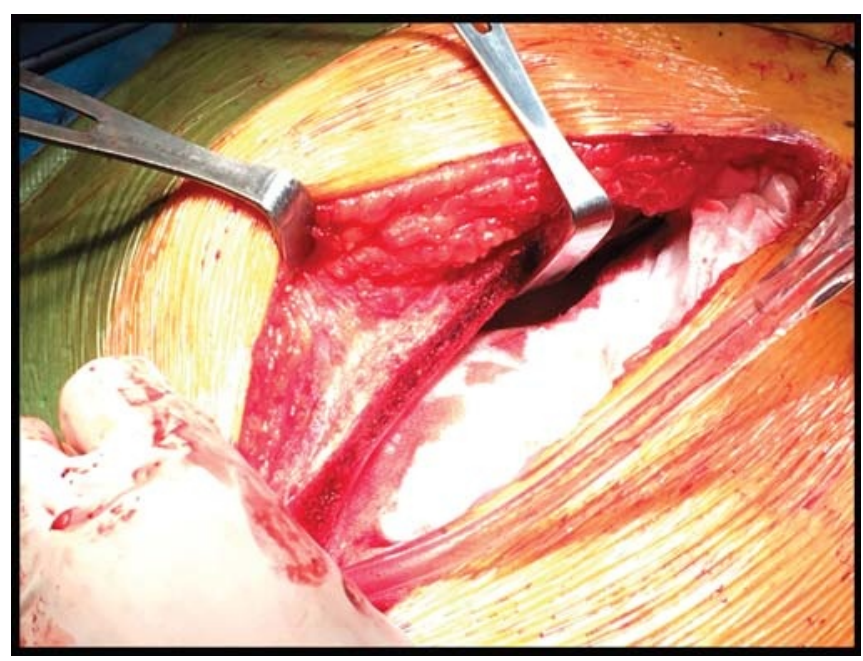

FIGURE 4. Sternum after removal of SPD.
After insertion of the IMA retractor in 6 patients, $5(83 \%)$ had no sternal bleeding while the IMA retractor was in place and $1(17 \%)$ of 6 patients had mild sternal bleeding while the IMA retractor was in place.

After insertion of the sternal retractor, $8(67 \%)$ of 12 patients had no sternal bleeding at 5 minutes, $3(25 \%)$ of 12 patients had mild sternal bleeding at 5 minutes, and only $1(8 \%)$ of 12 patients had moderate sternal bleeding at 5 minutes. At 30 minutes after insertion of the sternal retractor, $10(83 \%)$ of 12 patients had no sternal bleeding and $2(17 \%)$ of 12 patients had mild sternal bleeding.

Bleeding was seen to increase after removal of the SPD with $5(42 \%)$ of 12 patients having mild sternal bleeding, $3(25 \%)$ of 12 patients having moderate sternal bleeding, and $4(33 \%)$ of 12 patients having severe sternal bleeding.

Sternal trauma was assessed within 5 minutes of SPD removal. No visible sternal trauma was observed for any patient. Appearance of hemisternal section after removal of the SPD is shown in Figure 4.

Insertion of the device was assessed as "very easy" or "easy" in $8(67 \%)$ of 12 patients and removal was assessed as very easy or easy in all 12 patients $(100 \%)$ (Table 2$)$.

There was some difficulty with insertion of the IMA retractor with the SPD in situ with surgeons reporting that the IMA retractor was "neither easy nor difficult" to insert in $5(56 \%)$ of 9 patients and "difficult" or "very difficult" to insert in $4(44 \%)$ of 9 patients.

TABLE 2. Ease of Use Assessment

\begin{tabular}{|c|c|c|c|c|}
\hline \multirow[b]{2}{*}{ Scale } & \multicolumn{3}{|c|}{ Ease of Insertion } & \multirow{2}{*}{$\begin{array}{c}\text { Ease of Removal } \\
\text { SPD From } \\
\text { Hemisternal } \\
\text { Sections } \\
(\mathrm{N}=12)\end{array}$} \\
\hline & $\begin{array}{c}\text { SPD } \\
(N=12)\end{array}$ & $\begin{array}{l}\text { IMA Retractor } \\
\text { When SPD In } \\
\text { Situ }(n=9)\end{array}$ & $\begin{array}{c}\text { Sternotomy } \\
\text { Retractor When } \\
\text { SPD In Situ } \\
(\mathrm{N}=12)\end{array}$ & \\
\hline Very easy & 2 & - & 3 & 7 \\
\hline Easy & 6 & - & 2 & 5 \\
\hline $\begin{array}{l}\text { Neither easy } \\
\text { or difficult }\end{array}$ & 3 & 5 & 7 & - \\
\hline Difficult & 1 & 2 & - & - \\
\hline Very difficult & - & 2 & - & - \\
\hline
\end{tabular}

IMA, internal mammary artery; SPD, sternal protection device. 
TABLE 3. Overall Satisfaction Assessment

\begin{tabular}{|c|c|c|}
\hline Scale & No. Patients $(n=12)$ & Comments \\
\hline Very much satisfied & 2 & - \\
\hline Somewhat satisfied & 6 & - \\
\hline \multirow[t]{4}{*}{ Slightly satisfied } & 4 & $\begin{array}{l}\text { A bit 'fiddly' to put in and more } \\
\text { bleeding once removed. }\end{array}$ \\
\hline & & $\begin{array}{l}\text { Fiddly to use with the mammary } \\
\text { retractor. }\end{array}$ \\
\hline & & $\begin{array}{l}\text { Patient overweight and chest deep } \\
\text { making more difficult. }\end{array}$ \\
\hline & & $\begin{array}{l}\text { Patient was not wet and therefore } \\
\text { felt device was not needed. } \\
\text { Device made surgery more } \\
\text { difficult due to thin patient. }\end{array}$ \\
\hline
\end{tabular}

Not at all satisfied

Insertion of the sternal retractors with the SPD in situ was considered easy or very easy in $5(42 \%)$ of 12 patients and neither easy or difficult in 7 (58\%) of 12 patients.

Overall satisfaction (Global Impression) of the SPD was assessed immediately after surgery with two thirds of surgeons indicating "very much satisfied" and "somewhat satisfied" with the device (Table 3).

A total of 21 AEs were reported in 8 patients with most of AEs $(80 \%)$ mild in severity and not considered related to the device (Table 4). Three serious AEs reported in 2 patients were mild in severity and considered by the surgeon to be unrelated to the device (Table 5). Complete list of adverse events (Table 6, Supplemental Digital Content 2, http://links. lww.com/INNOV/A150).

Device deficiencies reported in 4 patients $(33 \%)$ were related to insertion and use of the IMA retractor when the device was in situ. In 3 patients (25\%), the device prevented the IMA retractor from holding onto the sternum resulting in slippage of the retractor. The device was removed and then reinserted before insertion of the sternal retractor. In 1 patient, the surgeon experienced difficulty in inserting the device and noted that the patient was very lean and the patient's skin hindered placement of the device.

At the 4-week follow-up assessment, there were no reports of sternal instability.

\section{DISCUSSION}

The primary objective of the study was to evaluate the safety of the Jupiter SPD during cardiac surgery. None of the AEs reported were considered to be related to the device, and the results provide initial data to support the safety of the device in cardiac surgery.

Secondary objectives of the study were to evaluate the ability of the device to protect the sternum from trauma, the ability of the

TABLE 4. Adverse Events

\begin{tabular}{lccc}
\hline & No. AEs $(\mathbf{N}=\mathbf{2 1})$ & No. Participants $(\mathbf{N}=\mathbf{1 2})$ \\
\hline \multirow{2}{*}{ Severity } & Mild & 17 & 7 \\
& Moderate & 4 & 3 \\
& Severe & - & -
\end{tabular}

AE, adverse event.
TABLE 5. Serious Adverse Events

\begin{tabular}{|c|c|c|c|c|c|}
\hline $\begin{array}{l}\text { Patient } \\
\text { No. }\end{array}$ & $\begin{array}{c}\text { SAE } \\
\text { Description }\end{array}$ & Severity & $\begin{array}{c}\text { Action } \\
\text { Taken } \\
\text { With } \\
\text { Device }\end{array}$ & $\begin{array}{c}\text { Therapy for } \\
\text { Event }\end{array}$ & $\begin{array}{c}\text { Seriousness } \\
\text { Criteria }\end{array}$ \\
\hline \multirow[t]{2}{*}{02} & Chest infection & Mild & None & Cephalexin & Hospitalization \\
\hline & $\begin{array}{l}\text { Right leg wound } \\
\text { infection }\end{array}$ & Mild & None & Cephalexin & Hospitalization \\
\hline 05 & Atrial flutter & Mild & None & Amiodarone & Hospitalization \\
\hline
\end{tabular}

SAE, serious adverse event.

device to reduce sternal bleeding during the period the device was in situ, and the ease of use of the device during cardiac surgery.

Because no trauma to the sternum was identified in any patients, in the absence of a control group, no conclusions can be drawn regarding the ability of the SPD to protect the sternum from trauma caused by sternal retractor blades.

All patients enrolled in the study were on anticoagulants/ antiplatelet drugs at the commencement of surgery. During the period the device was in situ, sternal bleeding was reduced in 9 of the 11 patients who had mild, moderate, or severe bleeding before insertion of the device.

The ease of use scores provide feedback on the design of the device. Overall, ease of use scores for the 2 types of sternal retractors used (Codman Ankeney Self Retaining Sternal Retractor and Codman Pilling Vascular Cardiovascular Morse Sternal Retractor) were equal to or less than 3 (scale: 1 = very easy to $5=$ very difficult). Ease of use scores for insertion of the Delacroix-Chevalier IMA Retractor were equal to or greater than 3. The IMA retractor was more difficult to insert when the device was used; the device caused slippage of the retractor in 3 patients and could not be inserted in 1 patient, limiting use of the device to 5 of 9 patients using the IMA retractor. The Delacroix-Chevalier IMA retractor is designed to avoid the need to connect the retractor to the side of the table. As a consequence, the retractor has blades that need to be placed over both hemisternal sections, and as the retractor is opened, the 2 sides are separated from each other with elevation of 1 side. This retractor is often difficult to insert under normal circumstances because of the limited space between the hemisternal sections.

Overall satisfaction (global impression) ranged from "slightly satisfied" to very much satisfied (scale: 1 = very much satisfied to $4=$ not at all satisfied); however, patient numbers were too small to draw any conclusions in relation to factors influencing the scores.

The results support the secondary objectives of the study. The reasons for slippage of the Delacroix-Chevalier IMA retractor and use of the device in thin patients require further investigation.

The study had a number of limitations. The administration of blood products was not recorded, and in the absence of a control group in this pilot study, it is not possible to confirm whether a reduction in hemoglobin was less than would have occurred in the absence of the device.

No enrolled patient reported a history of osteoporosis or other risk factors for fractures such as rheumatoid arthritis. In this study, data recorded on concomitant medications were limited and no information on use of drugs such as long-term corticosteroids was captured. Furthermore, patients at high risk of 
fracture were unlikely to be enrolled because of the age limitations ( $\geq 18$ and $\leq 80$ years). Thus, the individual or population risk profile for sternal trauma in this study is not known.

This open-label nonrandomized pilot study with 12 patients met the study objectives. The device was shown to be safe in the selected population and reduced bleeding was observed. The device was relatively easy to insert and remove although slippage was observed during IMA retractor insertion.

As a small study, the results are considered preliminary and further data are required from larger studies or in the postmarket setting to confirm the performance and safety of the device in a broader patient population including high-risk populations for sternal bleeding or sternal trauma.

\section{ACKNOWLEDGMENTS}

The authors thank Study Coordinators Margaret Quayle and Robyn Summerhayes, Study Monitor Anne DeLuca, Data Manager Helen Poliviou, and Protego Project Coordinator Ram Savana for their contribution to the study.

\section{REFERENCES}

1. Aigner P, Eskandary F, Schloglhöfer T, et al. Sternal force distribution during median sternotomy retraction. J Thorac Cardiovasc Surg. 2013; 146:1381-1386.
2. Bolotin G, Buckner GD, Campbell NB, et al. Tissue-disruptive forces during median sternotomy. Heart Surg Forum. 2007;10:487-492.

3. Moore R, Follette DM, Berkhoff HA. Poststernotomy fractures and pain management in open cardiac surgery. Chest. 1994;106: 1339-1342.

4. Kirbas A, Celik S, Gurer O, Yildiz Y, Isik O. Sternal wrapping for the prevention of sternal morbidity in elderly osteoporotic patients undergoing median sternotomy. Tex Heart Inst J. 2011;38:132-136.

5. Barnard J, Millner R. A review of topical hemostatic agents for use in cardiac surgery. Ann Thorac Surg. 2009;88:1377-1383.

6. Vestergaard RF, Jensen H, Vind-Kezunovic S, Jakobsen T, Søballe K, Hasenkam JM. Bone healing after median sternotomy: a comparison of two hemostatic devices. J Cardiothorac Surg. 2010;5: 117-117.

7. Aratari C, Manche A, Ferretti L, Fusella M. Cardiopulmonary bypass line sternal wrapping for protection and haemostasis. Interact Cardiovasc Thorac Surg. 2009;9:147-149.

8. Alberius P, Klinge B, Sjögren S. Effects of bone wax on rabbit cranial bone lesions. J Craniomaxillofac Surg. 1987;15:63-67.

9. Allison RT. Foreign body reactions and an associated histological artefact due to bone wax. Br J Biomed Sci. 1994;51:14-17.

10. Nelson DR, Buxton TB, Luu QN, Rissing JP. The promotional effect of bone wax on experimental Staphylococcus aureus osteomyelitis. J Thorac Cardiovasc Surg. 1990;99:977-980.

11. Mair H, Kaczmarek I, Oberhoffer M, et al. Surgicel Nu-Knit hemostat for bleeding control of fragile sternum. J Thorac Cardiovasc Surg. 2005;130: 605-606.

\section{CLINICAL PERSPECTIVE}

This is a study of a new device that is intended to protect the cut hemisternal edges from trauma and to reduce blood loss during open cardiac surgery. This was a small feasibility study in 12 patients. After sternotomy, the sternal protection device was placed over each hemisternal section before insertion of the retractor and remained in situ until the end of surgery. The device was generally considered easy to use. During surgery, the surgeon assessed and recorded sternal bleeding at six time points. After insertion, sternal bleeding was reduced in 9 of 11 patients who had mild or more bleeding before insertion of the device. There were no device-related adverse events. Generally, the device was easy to insert and most of the problems were related to its use with the internal mammary artery retractor.

In summary, this was a well-designed, small feasibility study. The study had a number of important limitations. There was no control group. Moreover, the determination of bleeding was subjective and not quantified. These were simply preliminary results and do not support either the safety or efficacy of this device at this point. Further studies in a larger population of patients will be needed to fully evaluate the device's ability to reduce the morbidity associated with sternal bleeding and sternal trauma in patients undergoing cardiac surgery. 